\title{
Gene and miRNA expression profiles in PBMCs from patients with severe and mild emphysema and PiZZ alpha I-antitrypsin deficiency
}

This article was published in the following Dove Press journal: International Journal of COPD

\author{
Cristina Esquinas ${ }^{1,2}$ \\ Sabina Janciauskiene ${ }^{3}$ \\ Ricardo Gonzalo 4 \\ Gemma Mas de Xaxars ${ }^{4}$ \\ Beata Olejnicka ${ }^{5}$ \\ Irene Belmonte 6 \\ Miriam Barrecheguren' \\ Esther Rodriguez' \\ Alexa Nuñez' \\ Francisco Rodriguez-Frias ${ }^{6}$ \\ Marc Miravitlles' \\ 'Pneumology Department, University \\ Hospital Vall d'Hebron, CIBER \\ de Enfermedades Respiratorias \\ (CIBERES), Barcelona, ${ }^{2}$ Public Health, \\ Mental, Maternal and Child Health \\ Nursing Department, Faculty of \\ Medicine and Health Sciences, \\ University of Barcelona, Barcelona, \\ Spain; ${ }^{3}$ Department of Respiratory \\ Medicine, Hannover Medical School, \\ BREATH, German Center for Lung \\ Research (DZL), Hannover, Germany; \\ ${ }^{4}$ Statistics and Bioinformatics Unit \\ (UEB), Vall d'Hebron Research \\ Institute (VHIR), Barcelona, Spain; \\ ${ }^{5}$ Department of Experimental Medical \\ Science, Lund University, Lund, \\ Sweden; ${ }^{6}$ Biochemistry Department, \\ University Hospital Vall d'Hebron, \\ Barcelona, Spain
}

Correspondence: Marc Miravitlles Servei de Pneumologia, Hospital Universitari Vall d'Hebron,

P Vall d'Hebron I19-129,

08035 Barcelona, Spain

Tel +34 932746157

Fax +34932746083

Email mmiravitlles@vhebron.net
Introduction: COPD has complex etiologies involving both genetic and environmental determinants. Among genetic determinants, the most recognized is a severe PiZZ (Glu342Lys) inherited alpha1-antitrypsin deficiency (AATD). Nonetheless, AATD patients present a heterogeneous clinical evolution, which has not been completely explained by sociodemographic or clinical factors. Here we performed the gene expression profiling of blood cells collected from mild and severe COPD patients with PiZZ AATD. Our aim was to identify differences in messenger RNA (mRNA) and microRNA (miRNA) expressions that may be associated with disease severity.

Materials and methods: Peripheral blood mononuclear cells from 12 COPD patients with PiZZ AATD (6 with severe disease and 6 with mild disease) were used in this pilot, highthroughput microarray study. We compared the cellular expression levels of RNA and miRNA of the 2 groups, and performed functional and enrichment analyses using the Kyoto Encyclopedia of Genes and Genomes (KEGG) and Gene-ontology (GO) terms. We also integrated the miRNA and the differentially expressed putative target mRNA. For data analyses, we used the R statistical language R Studio (version 3.2.5).

Results: The severe and mild COPD-AATD groups were similar in terms of age, gender, exacerbations, comorbidities, and use of augmentation therapy. In severe COPD-AATD patients, we found 205 differentially expressed genes (DEGs) (114 upregulated and 91 downregulated) and 28 miRNA (20 upregulated and 8 downregulated) compared to patients with mild COPDAATD disease. Of these, hsa-miR-335-5p was downregulated and 12 target genes were involved in cytokine signaling, MAPK/mk2, JNK signaling cascades, and angiogenesis were much more highly expressed in severe compared with mild patients.

Conclusions: Despite the small sample size, we identified downregulated miRNA (hsa-miR$335)$ and the activation of pathways related to inflammation and angiogenesis on comparing patients with severe vs mild COPD-AATD. Nonetheless, our findings warrant further validation in large studies.

Keywords: alpha1-antitrypsin deficiency, COPD, gene expression, miRNAs, integrative analysis

\section{Introduction}

COPD is the third leading cause of death worldwide. It is a complex disease influenced by both genetic and environmental determinants, the most important of which is cigarette smoking. However, only $10 \%-20 \%$ of smokers develop clinically significant disease, and there is a significant variability across the smokers with similar cigarette exposure. Some of these heterogeneities may be the result of genetic variations. ${ }^{1}$ 
A known genetic risk factor for COPD is severe alpha1-antitrypsin deficiency (AATD). SERPINA1 is the gene responsible for the synthesis of the AAT protein, which is a serine protease inhibitor (serpin) and an acute-phase glycoprotein. ${ }^{2}$ Normal serum AAT levels are the result of 2 copies of the common $M$ allele of the SERPINA1 gene. The M allele of AAT is present in $>90 \%$ of the population, and is called Pi*M. Over 100 SERPINA1 mutations have been identified to date, but only some are implicated in disease pathogenesis. The most frequent deficient alleles are $\mathrm{S}$ (Glu264Val) and Z (Glu342Lys), which are called Pi*S and $\mathrm{Pi}^{*} \mathrm{Z}$, respectively. In particular, carriers of $2 \mathrm{Z}$ alleles (PiZZ) have strongly reduced circulating levels of AAT (up to $10 \%-20 \%$ of normal, which is $1.3-2 \mathrm{~g} / \mathrm{L}$ ). Because of $Z$ AAT misfolding and intracellular accumulation, the $\mathrm{Z}$ allele is the major contributor to pulmonary emphysema and liver disease in people of European ancestry. ${ }^{3-4}$

The evolution of emphysema in clinical terms and life expectancy are directly related to the accelerated loss of forced expiratory volume in $1 \mathrm{~s}\left(\mathrm{FEV}_{1}\right)$. Previous studies identified tobacco consumption and exacerbations as the main factors related to $\mathrm{FEV}_{1}$ decline. ${ }^{5}$ Lung function decline is faster in AATD compared with non-AATD COPD patients, although this is not homogeneous in all individuals. ${ }^{6-8}$

Recent genome-wide association studies (GWAS) and meta-analyses of GWAS have begun to shed light on biological pathways that contribute to the development of COPD. ${ }^{9-11}$ The analysis of the miRNA expression is increasingly used in clinical research. miRNAs belong to the family of small noncoding RNAs ( $\sim 21-25$ nucleotides long), but they are involved in mRNA regulation. ${ }^{12}$ miRNAs modulate gene expression by either destabilizing transcripts or inhibiting protein translation. In fact, miRNAs may target up to one-third of the transcriptome. Therefore, miRNA expression patterns in cells and tissues are considered to be biomarkers for both the diagnosis and prognosis of different diseases, including COPD. ${ }^{13-16}$ mRNA and miRNA signatures can assist clinicians in prognosis assessment and therapeutic approaches. ${ }^{12-18}$ However, the heterogeneity in study designs makes comparisons across studies difficult. Therefore, this field is naturally progressing toward the integration of miRNA with gene expression. ${ }^{19}$

In this context, some SERPINA1 gene expression studies have been performed. ${ }^{6}$ We propose that gene expression profiling may provide new insights into the molecular mechanisms of AATD-related COPD and eventually lead to the identification of novel therapeutic targets. ${ }^{20-21}$ Here, we explored the use of gene expression patterns in peripheral blood mononuclear cells (PBMCs) collected from PiZZ cases with severe and mild
COPD. We aimed to identify differentially expressed putative mRNA and miRNAs in PBMCs isolated from patients with different severities of lung disease.

\section{Materials and methods Design and study sample}

This study was a pilot high-throughput microarray experiment. On the basis of the presence of poor lung function at diagnosis $\left(\mathrm{FEV}_{1}<45 \%\right.$ predicted), we included 6 patients considered to have rapid progression of emphysema (severe disease group). This severe group was compared with a group of 6 subjects matched for age and gender but with significantly better lung function at diagnosis $\left(\mathrm{FEV}_{1}>65 \%\right.$ predicted). We considered these patients to have slow disease progression (mild disease group). All the patients were recruited from the outpatient clinic of the respiratory department of the Vall d'Hebron University Hospital (Barcelona, Spain) and all had undergone genotyping by sequencing of all coding exons of SERPINA1. Signed informed consent was obtained from all subjects for participation in the study, and the study was approved (project ID: PR (AG)157/2016) by the Ethics Committee of the Research Institute of Hospital Vall d'Hebron (Barcelona, Spain).

All the patients presented clinical stability, defined as no respiratory ambulatory exacerbations or hospital admission in the previous 2 months. They were followed and treated according to guidelines. ${ }^{22}$ Some patients were receiving intravenous therapy with plasma purified AAT (Prolastin or Trypsone; Grifols, Barcelona, Spain) at a dose of $120 \mathrm{mg} / \mathrm{kg}$ for 15 days.

As previous studies have shown that AAT therapy may indirectly reduce the expression of Z-AAT, ${ }^{23}$ blood samples were taken immediately before the next infusion in patients receiving augmentation therapy.

\section{Variables and data collection}

Sociodemographic, clinical (phenotype, age at diagnosis, current age, age at onset of symptoms, tobacco consumption, comorbidities, exacerbations, and treatments), and lung function data at the time of AATD diagnosis were collected from medical records. Lung function tests were performed according to standardized recommendations and international guidelines. ${ }^{24-25}$

\section{Blood processing and RNA isolation}

Blood processing and RNA isolation was carried out at the Biobank at the Vall d'Hebron Research Institute (VHIR), Barcelona, Spain. PBMCs were isolated from AATD patients using Ficoll gradient centrifugation. Total RNA and miRNA were isolated from PBMCs using the QuickGene RNA tissue 
SII (RT-S2) kit and QuickGene 810 FUJIFILM device. miRNA was also isolated from PBMCs using QIAzol Lysis Reagent (Qiagen, Hilden, Germany) and the miRNeasy Mini Handnook Kit (Qiagen). RNA and miRNA concentrations were determined with a NanoDrop ND2000 spectrophotometer and quality was checked using a Bioanalyzer (Agilent Technologies, Hilden, Germany) with Eukariotic NanoChip before microarray analysis.

\section{RNA and miRNA microarray hybridization}

The RNA and miRNA microarrays were performed at the High Technology Unit (UAT) of the VHIR. In this experiment, the Affymetrix GeneTitan microarray platform and the Genechip Human Gene Array Plate 2.1 were used for RNA hybridization. This array analyzes gene expression patterns on a wholegenome scale on a single array with probes covering many exons on the target genomes, thereby providing expression summarization at the exon or gene level. The starting material was 200 ng of total RNA of each sample. Briefly, sense singlestranded DNA (ssDNA) suitable for labeling was generated from total RNA with the GeneChip WT Plus Reagent Kit from Affymetrix (High Wycomb, UK) according to the manufacturer's instructions. Sense ssDNA was fragmented, labeled, and hybridized to the arrays with the GeneChip WT Terminal Labeling and Hybridization Kit from the same manufacturer.

The Affymetrix GeneTitan microarray platform and the Genechip miRNA array plate 4.1 were used for miRNA hybridization. The starting material was $400 \mathrm{ng}$ of total RNA of each sample. miRNA in the sample was labeled using the Flash Tag Biotin HSR RNA Labeling Kit following the manufacturer's instructions.

We used a unique miRNA and 2 mRNA microarray batches in our experiments. Distribution of severe and mild patient samples within the batches was similar.

\section{Statistical analysis for comparison of patient characteristics}

The results of the statistical analyses are expressed as mean (SD) for quantitative variables and as valid percentage for groups using the nonparametric Mann-Whitney $U$ or chisquare tests (Fisher's exact test when the expected frequencies were $<5)$.

\section{Data preprocessing and analysis of differentially expressed genes}

First, a raw data quality control was performed before normalization in order to analyze sample quality, hybridization quality, signal comparability and biases, array correlation, and probe sets' homogeneity.

Background correction and quantile normalization were performed for the raw array data using the oligo package (http://www.bioconductor.org/) ${ }^{26}$ and robust multi-array average (RMA package for R). ${ }^{27}$ For each sample, the expression values of all probes for a given gene were reduced to a single value by taking the average expression value. Principal component analysis (PCA) was performed and a hierarchical clustering plot was made after normalization. Batch effects were analyzed using surrogate variable analysis (SVA package ${ }^{28}$ and Combining Batches of Gene Expression Microarray Data (ComBat) functions. ${ }^{29}$

Before differentially expressed genes (DEGs) analysis, present probes without the corresponding gene symbols and expression intensities below the 3rd quartile value were removed for further statistical analysis. The Limma (Linear Models for Microarray Data) package ${ }^{30}$ was used to perform statistical testing of differential mRNA and miRNA expression between the 2 study groups. Differentially expressed mRNAs and miRNAs were defined as having a $P$-value of $<0.05$ with at least \pm 1.5 -fold change (FC) $(\log \mathrm{FC} \pm 0.58)$ between the groups. $P$-values adjusted for the false discovery rate (FDR) were also calculated.

\section{DEGs annotation and pathway- enrichment analyses}

DEGs were annotated identifying the beginning and the end of the chromosome as well as the gene symbol with the BioMart R package (http://www.biomart.org) and the Affymetrix annotation files.

Kyoto Encyclopedia of Genes and Genomes (KEGG) and Gene-ontology (GO) terms ${ }^{31}$ were enriched for DEGs using the Gene Expression Omnibus (https://www.ncbi.nlm. nih.gov/geo/) database. The major GO terms associated with biological processes was manually summarized based on gene-term enrichment provided for each functional group at a Fisher's exact $P$-value of 0.05 . The same statistical approach was used for KEGG analysis.

\section{miRNA and mRNA data integration}

Integration of miRNA DEGs and their target mRNA DEGs was performed using the multiMiR $\mathrm{R}$ package with the web server at http://multimir.ucdenver.edu. This is a comprehensive collection of predicted and validated miRNAtarget interactions and their associations with diseases and drugs. ${ }^{32}$ All the analyses were performed using the R Studio (version 3.2.5). 


\section{Quantitative reverse transcription polymerase chain reaction validation}

For all the samples, an independent analysis of EGR3 and EREG expression was performed by the quantitative reverse transcription polymerase chain reaction (qRT-PCR) method. The levels of expression of selected genes were analyzed using the TaqMan Gene Expression Assay (Applied Biosystems, Life Technologies, Madrid, Spain). The expression of the reference gene, hypoxanthine phosphoribosyl transferase (Life Technologies, \#Hs02800695_m1), was used for normalization. Relative gene expression was calculated according to the $\Delta$ cycle threshold method using StepOne software. Statistical significance was defined as $P<0.05$ as measured using the Student's $t$-test.

\section{Results}

Blood PBMCs were isolated from 12 PiZZ patients: 6 with severe emphysema with a mean $\mathrm{FEV}_{1}(\%)$ of $44 \%(\mathrm{SD}=6.7 \%)$ and 6 with mild disease, with $\mathrm{FEV}_{1}(\%)$ of $93 \%$ (SD $=29 \%$ ). Both study groups presented similar characteristics in terms of age, gender, exacerbations, comorbidities, and time from the last infusion. Severe patients were diagnosed at a younger age than mild patients (42.7 years [SD $=6.4$ ] vs $51.2[\mathrm{SD}=4.0]$, $P=0.041$ ) and all received inhaled corticosteroids (Table 1).

Table I Patients' characteristics

\begin{tabular}{|c|c|c|c|}
\hline Variables & $\begin{array}{l}\text { Mild } \\
\text { emphysema } \\
\mathbf{N}=6\end{array}$ & $\begin{array}{l}\text { Severe } \\
\text { emphysema } \\
\mathbf{N}=6\end{array}$ & $P$-value \\
\hline $\begin{array}{l}\text { Age (years) at the time of the } \\
\text { study }\end{array}$ & $61.7(7.7)$ & $61.0(10.5)$ & 0.937 \\
\hline Age (years) at diagnosis & $51.2(4)$ & $42.7(6.4)$ & 0.041 \\
\hline Gender & & & 0.999 \\
\hline Male (\%) & $4(66.7)$ & $4(66.7)$ & \\
\hline Female (\%) & $2(33.3)$ & $2(33.3)$ & \\
\hline Smoking history & & & 0.135 \\
\hline Never (\%) & $2(33.3)$ & 0 & \\
\hline Current (\%) & 0 & $2(33.3)$ & \\
\hline Former (\%) & $4(66.7)$ & $4(66.7)$ & \\
\hline $\mathrm{FEV}, \%$ & $93(29)$ & $44(6.7)$ & 0.002 \\
\hline AAT blood levels (mg/dL) & $22(5)$ & $27(5.1)$ & 0.265 \\
\hline \multicolumn{4}{|l|}{ Other comorbidities } \\
\hline Bronchiectasis & I (I6.7) & I (16.7) & 0.098 \\
\hline Cardiovascular diseases & I (16.7) & $2(33.3)$ & 0.125 \\
\hline $\begin{array}{l}\text { Inhaled respiratory } \\
\text { treatments (\%) }\end{array}$ & $4(66.7)$ & $6(100)$ & 0.225 \\
\hline Beta2 agonists (\%) & $4(66.7)$ & $6(100)$ & 0.065 \\
\hline Inhaled corticosteroids (\%) & 0 & $6(100)$ & 0.025 \\
\hline Replacement therapy (RT) (\%) & $2(33.3)$ & $4(66.7)$ & 0.248 \\
\hline $\begin{array}{l}\text { Time from last RT } \\
\text { infusion (days) }\end{array}$ & 15 & 15 & 0.999 \\
\hline $\begin{array}{l}\text { Time from blood collection } \\
\text { to sample process (minutes) }\end{array}$ & $74.2(49.6)$ & $68.3(23.2)$ & 0.589 \\
\hline
\end{tabular}

Notes: Data are expressed as mean (SD) or $\mathrm{n}$ (valid percentage). Bold values indicate $p<0.05$.

Abbreviations: AAT, alphal-antitrypsin; $\mathrm{FEV}_{1}$, forced expiratory volume in I s.
Two patients from the severe group were current smokers and, therefore, did not receive replacement therapy. None of the patients in either group received systemic corticosteroids. The time from blood collection and processing in the laboratory was compared between groups without statistical differences.

\section{mRNA analysis}

Initially, the mRNA expression of 53,617 probe sets corresponding to 12 samples were included. Data were normalized and after filtering, a total of 10,143 probes were used for the following analysis. We found no batch effects according to the time of blood processing, study group, or gender (data not shown).

Normalized PCA results showed that the first axis explained $16 \%$ of the variance. Two samples corresponding to current smokers from the severe group presented a different gene expression profile according to the second axis that explained $10.5 \%$ of the variability. To avoid a potential gene expression bias, these 2 samples were removed from the following analyses. Finally, 10 AATD samples (4 from the severe and 6 from the mild disease groups) were included in the DEGs analysis.

A total of 205 DEGs differed between severe and mild patients, of which 114 genes were upregulated and 91 downregulated in severe patients using non-adjusted $P$-value of $<0.05$ and $\mathrm{FCs}> \pm 1.5(\log \mathrm{FC}> \pm 0.58)$ (Figure 1$)$.

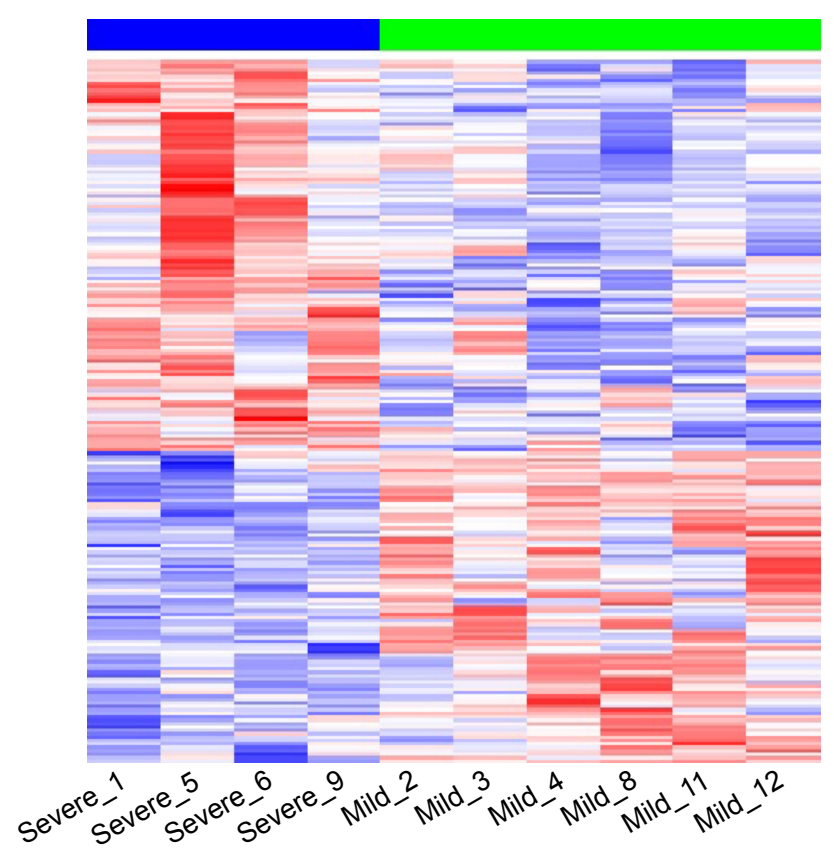

Figure I Differentially expressed genes in severe (blue line) vs mild (green line) patients.

Notes: Two-dimensional hierarchical clustering of the 10 samples according to the expression of 205 genes differentially expressed (by row) comparing severe $(n=4)$ vs mild ( $n=6$ ) patients (by column), with a $P$-value of $<0.05$ and fold changes (FC) $> \pm 1.5$ ( $\log F C> \pm 0.58)$ using the Limma (Linear Models for Microarray Data) method. The functional classification of genes (color-coded) is shown. Red represents a high level of gene expression while blue represents a low level of expression. 
FDR $P$-values were calculated, but no differences were found in gene expression between the 2 groups. Therefore, for all the following analyses, we used non-adjusted $P$-values.

The top dysregulated mRNAs are shown in Table 2. Upregulated genes with highest difference between the 2 groups $(\operatorname{logFC}>1)$ included HLA-DRB5, OLFM4, HBEGF, LFT, MMP8, CXCL8, SOCS3, CAMP, G0S2, CCL3L3, EREG, EGR3, NR4A2, JUP, and IL1B genes.

These genes are involved in systemic and local inflammation, immunity, proliferation, differentiation, apoptosis, and oncogenesis. We found that these groups of genes are enriched in different pathways such as the mitogen-activated protein kinases (MAPK) cascade pathway, the Jun aminoterminal kinases (JNK) cascade, the Janus kinase/signal transducers and activators of transcription (JAK/STAT) signaling pathway, the NOD-like receptor pathway, and the Toll-like receptor signaling pathway using GO and KEGG terms (Tables 3 and 4).

Table 2 Top dysregulated mRNA comparisons in peripheral blood mononuclear cells from severe vs mild AATD emphysema patients

\begin{tabular}{|c|c|c|}
\hline Symbols & $\log \mathrm{FC}$ & $P$-value \\
\hline \multicolumn{3}{|l|}{ Upregulated } \\
\hline HLA-DRB5 & 2.46 & 0.025 \\
\hline OLFM4 & 2.42 & 0.003 \\
\hline HBEGF & 2.01 & 0.002 \\
\hline LTF & 1.79 & 0.047 \\
\hline MMP8 & 1.69 & 0.028 \\
\hline CXCL8/LL-8 & 1.57 & 0.019 \\
\hline SOCS3 & 1.56 & 0.000 \\
\hline CAMP & 1.53 & 0.014 \\
\hline GoS2 & 1.50 & 0.015 \\
\hline CCL3L3/CCL3LI & 1.38 & 0.001 \\
\hline EREG & 1.37 & 0.018 \\
\hline EGR3 & 1.31 & 0.004 \\
\hline NR4A2 & 1.31 & 0.004 \\
\hline OSM & 1.28 & 0.007 \\
\hline CRISP3 & 1.24 & 0.043 \\
\hline ILIB & 1.18 & 0.014 \\
\hline JUP & 1.16 & 0.008 \\
\hline PTGS2 & 1.11 & 0.038 \\
\hline IDI & 1.11 & 0.003 \\
\hline CLEC5A & 1.10 & 0.003 \\
\hline NR4A3 & 1.08 & 0.027 \\
\hline SNAII & 1.00 & 0.003 \\
\hline $\mathrm{CD} 24$ & 1.00 & 0.034 \\
\hline \multicolumn{3}{|l|}{ Downregulated } \\
\hline$X C L 2$ & -1.01 & 0.000 \\
\hline PDK4 & -1.01 & 0.047 \\
\hline RNUI-59P & -1.05 & 0.013 \\
\hline ZNF208 & -1.12 & 0.000 \\
\hline
\end{tabular}

Note: Table 2 shows different miRNAs using a $P$-value $<0.05$ and $\log F C> \pm$ I. Abbreviations: AATD, alphal-antitrypsin deficiency; IL-8, interleukin-8; logFC, fold change logarithm; MMP, matrix metalloproteases protease; mRNA, messenger RNA.

\section{miRNA DEGs analysis}

The miRNA data were analyzed using the same pipeline as in mRNA data analysis. Initially, a quality control of 6,658 miRNA probes was performed and after filtering, 1,165 miRNA were included in the Limma analysis. A total of 28 miRNAs were found to be differently expressed in PBMCs from severe compared to mild AATD emphysema patients, with 20 miRNAs being upregulated and 8 being downregulated in severe patients (Table 5).

All the dysregulated miRNAs are shown in Table 5. Upregulated miRNA included hsa-miR-146b-3p, hsa-miR146b-5p, hsa-miR-193b-3p, and hsa-miR-365a-5p, whereas downregulated miRNAs included hsa-miR-486-3p and hsamiR-335-5p. The latter 2 have previously been reported to be related to respiratory diseases.

\section{miRNA and mRNA interactions}

As shown in Table 6, we identified 3 putative miRNA interacting targets between differentially expressed miRNAs and DEGs. Our analysis showed significant downregulation of hsa-miR-335-5p in severe patients, which was significantly linked to 12 upregulated transcripts, enriched in the cytokinemediated signaling pathway, the MAPK/mk2 signaling cascade, the JNK cascade, and angiogenesis (Table 6).

\section{qRT-PCR validation of DEGs}

We randomly selected EREG and EGR3 genes to be verified by qRT-PCR. We have chosen these 2 genes with lower FCs, hoping to reproduce our findings by using an independent method. In accordance with microarray data, the expression of both genes was significantly higher in PBMCs from severe than from mild AATD emphysema patients (Figure 2).

\section{Discussion}

One of the important issues in developing biomarkers for clinical purposes is the selection of appropriate cells or tissues from which candidates can be identified. Because of the systemic nature of COPD/emphysema ${ }^{33}$ and because of unique properties of AAT protein, ${ }^{34}$ we hypothesized that genome-wide expression profiling of peripheral blood cells may be used to identify signatures of AATD-related emphysema phenotypes. PBMCs are attractive candidates because they are easy to obtain and simple to prepare. Therefore, here we performed the gene expression profiling of PBMCs collected from severe and mild emphysema patients with PiZZ AATD. Our objective was to identify putative differences in mRNA and miRNAs expression that might be associated with disease severity. The microarray analysis of PBMCs isolated from 10 emphysema patients with 
Table 3 Gene ontology functional enrichment analysis of the DEGs between peripheral blood mononuclear cells isolated from severe vs mild AATD emphysema patients

\begin{tabular}{|c|c|c|c|c|}
\hline GO ID & Term & Odds ratio & $P$-value & DEGs \\
\hline \multicolumn{5}{|l|}{ Upregulated } \\
\hline 0043408 & Regulation of MAPK cascade & 4.8 & $9.03 e-07$ & $\begin{array}{l}\text { ATF3, CCRI, HBEGF, DUSPI, EREG, IDI, ILIB, OSM, } \\
\text { PARK2, TPD52LI, VEGFA, WNT7A, ARHGEF5, TRIBI, } \\
\text { DACTI, AVPII, CARD9, DUSPI6, CCL3L3, CD24 }\end{array}$ \\
\hline 0001525 & Angiogenesis & 4.68 & $4.4 \mid \mathrm{e}-05$ & $\begin{array}{l}\text { ADM, ANPEP, EGR3, EREG, NR4AI, IDI, ILIB, CXCL8, } \\
\text { MFGE8, PTGS2, VEGFA, WNT7A, PLXDCI }\end{array}$ \\
\hline 0001817 & Regulation of cytokine production & 4.17 & $7.3 e-05$ & $\begin{array}{l}\text { CAMP, CDI4, CLC, EREG, HLA-DRB5, ILIB, LTF, PTGS2, } \\
\text { NR4A3, SOCSI, CLEC5A, CARD9, ATG9A, CD24 }\end{array}$ \\
\hline 0007254 & JNK cascade & 6.17 & $9.2 \mathrm{e}-05$ & $\begin{array}{l}\text { ILIB, PARK2, TPD52LI, WNT7A, ARHGEF5, TRIBI, } \\
\text { DACTI, CARD } 9\end{array}$ \\
\hline 001937 & Cyclooxygenase pathway & 39.6 & 0.00012 & PTGDS, PTGS2, HPGDS \\
\hline 0019221 & Cytokine-mediated signaling pathway & 3.55 & 0.00019 & $\begin{array}{l}\text { CCRI, EGRI, EREG, CXCL2, HLA-DRB5, ILIB, CXCL8, } \\
\text { OSM, PARK2, SOCSI, SOCS3, CCL3L3, CD24 }\end{array}$ \\
\hline 0007249 & I-kappaB kinase/NFKB signaling & 4.51 & 0.00068 & CDI4, ILIB, LTF, PARK2, PTGS2, OLFM4, PLK2, CARD9 \\
\hline \multicolumn{5}{|c|}{ Downregulated } \\
\hline 0043949 & Regulation of cAMP-mediated signaling & 25.7 & 0.00345 & CXCLI0, PTGIR \\
\hline 0035490 & $\begin{array}{l}\text { Regulation of leukotriene production } \\
\text { involved in inflammatory response }\end{array}$ & $\operatorname{lnf}$ & 0.00382 & SERPINEI \\
\hline 0055118 & $\begin{array}{l}\text { Negative regulation of cardiac muscle } \\
\text { contraction }\end{array}$ & 265 & 0.00762 & PDESA \\
\hline 0007599 & Hemostasis & 3.75 & 0.0034 & A2M, SERPINEI, PTGIR, TFPI, PDE5A, P2RYI2, HIST2H3D \\
\hline 0006950 & Response to stress & 2.44 & 0.000726 & $\begin{array}{l}\text { A2M, HLA-DQBI, HLA-DRBI, IGFBP4, CXCLI0, OAS3, } \\
\text { SERPINEI, PDK4, PTGIR, XCL2, TFPI, HISTIH2BG, } \\
\text { HISTIH2BF, HIST2H2BE, PDE5A, MTSSI, DCLREIA, } \\
\text { PDCD6, KLRGI, CRTAM, P2RYI2, TNIP3, ZKSCAN3, } \\
\text { DUSPI9, HIST2H3D, GTF2H2C_2 }\end{array}$ \\
\hline
\end{tabular}

Abbreviations: AATD, alphal-antitrypsin deficiency; cAMP, cyclic adenosine monophosphate; DEGs, differentially expressed genes; GO, gene ontology; JNK, jun aminoterminal kinases; MAPK, mitogen-activated protein kinases; NFKB, nuclear factor kappa B.

PiZZ AATD revealed that cells from patients with severe disease presented 205 differentially expressed mRNAs (114 upregulated and 91 downregulated) and 28 miRNAs (20 upregulated and 8 downregulated) compared to those from patients with mild disease. Moreover, we found an interaction between downregulated hsa-miR-335-5p and 12 upregulated target gene mRNAs. These latter genes are related to the cytokine-mediated signaling pathway, the MAPK/mk2 signaling cascade, the JNK cascade, and angiogenesis. It is important to remark that all our patients were clinically stable and took their usual respiratory treatment at inclusion in the study.

In the cells of severe patients, we found a higher expression of the EREG, EGR3, and TRIB1 genes, which are involved in inflammatory response-related signal transduction pathways including MAPK. ${ }^{35}$ EGR3 is a zinc finger transcription factor, an immediate-early response protein activated by cellular stressors. ${ }^{36}$ As a major transcription factor, EGR3 alters the expression of several target genes, including repair enzyme systems, angiogenic factors, cytokines (interleukin-8 [IL-8]

Table 4 KEGG functional enrichment analysis of the DEGs between peripheral blood mononuclear cells isolated from severe vs mild AATD emphysema patients

\begin{tabular}{lllll}
\hline KEGG ID & Term & Odds ratio & P-value & DEGs \\
\hline 04640 & Hematopoietic cell lineage & 5.7 & 0.0014 & ANPEP, CDI4, HLA-DRBI, HLA-DRB5, ILIB, CD24 \\
00590 & Arachidonic acid metabolism & 5.9 & 0.00654 & PTGDS, PTGS2, HPGDS, PLA2G2C \\
04621 & NOD-like receptor pathway & 5.9 & 0.00625 & CXCL2, ILIB, CXCL8, CARD9 \\
04060 & Cytokine-cytokine receptor interaction & 2.89 & 0.00786 & CCRI, CXCL2, ILIB, CXCL8, OSM, XCL2, VEGFA, CCL3L3 \\
05310 & Asthma & 8.29 & 0.00769 & HLA-DQBI, HLA-DRBI, HLA-DRB5 \\
04062 & Chemokine signaling pathway & 2.69 & 0.036 & CCRI, CXCL2, CXCL8, CXCLI0, XCL2, CCL3L3 \\
04620 & Toll-like receptor signaling pathway & 3.14 & 0.046 & CDI4, ILIB, CXCL8, CXCLI0 \\
\hline
\end{tabular}

Abbreviations: AATD, alphal-antitrypsin deficiency; DEGs, differentially expressed genes; KEGG, Kyoto Encyclopedia of Genes and Genomes; NOD, nucleotide-binding oligomerization domain. 
Table 5 Dysregulated miRNA in peripheral blood mononuclear cells from severe compared with mild AATD emphysema patients

\begin{tabular}{lll}
\hline miRNA ID & logFC & $P$-value \\
\hline Upregulated & & \\
hsa-miR-708-5p & 2.46 & 0.009 \\
hsa-miR-4440 & 2.42 & 0.007 \\
hsa-miR-193b-3p & 2.01 & 0.036 \\
hsa-miR-3934-5p & 1.79 & 0.030 \\
hsa-miR-7846-3p & 1.69 & 0.003 \\
hsa-miR-873-5p & 1.57 & 0.008 \\
hsa-miR-27a-5p & 1.56 & 0.003 \\
hsa-miR-3064-3p & 1.53 & 0.018 \\
hsa-miR-146b-3p & 1.50 & 0.003 \\
hsa-miR-193b-5p & 1.38 & 0.009 \\
hsa-miR-873-3p & 1.37 & 0.035 \\
hsa-miR-146b-5p & 1.31 & 0.006 \\
hsa-miR-2278 & 1.31 & 0.021 \\
hsa-miR-1255a & 1.28 & 0.005 \\
hsa-miR-1225-5p & 1.24 & 0.035 \\
hsa-miR-135a-3p & 1.18 & 0.004 \\
hsa-miR-4723-5p & 1.16 & 0.037 \\
hsa-miR-874-3p & 1.11 & 0.008 \\
hsa-miR-365a-5p & 1.11 & 0.003 \\
hsa-miR-29b-I-5p & 1.10 & 0.003 \\
Downregulated & & \\
hsa-miR-543 & -1.00 & 0.018 \\
hsa-miR-139-3p & -1.00 & 0.013 \\
hsa-miR-485-3p & -1.02 & 0.049 \\
hsa-miR-335-5p & -1.01 & 0.047 \\
hsa-miR-139-5p & -1.01 & 0.040 \\
hsa-miR-486-3p & -1.05 & 0.049 \\
hsa-miR-185-3p & -1.12 & 0.020 \\
hsa-miR-328-3p & -1.20 & 0.025 \\
\hline Note:Tale5 shows & \\
\hline
\end{tabular}

Note: Table 5 shows different miRNAs using $P$-value $<0.05$ and $\operatorname{logFC}> \pm 1$. Abbreviations: AATD, alpha I-antitrypsin deficiency; logFC, fold change logarithm; miRNA, microRNA.

and tumor necrosis factor-alpha $[\mathrm{TNF} \alpha]$ ), apoptotic factors (Fas), cell cycle factors (p21 and p53), metabolic factors, and matrix metalloproteases proteases (MMPs). In parallel to EGR3, we also found a higher expression of cytokines (IL1B, CCL3L3, and CXCL8). Hence, there may be a direct link between EGR3 and cytokine expression, which has already been validated as a target gene in respiratory inflammation and different cancers. ${ }^{37}$

The activation of members of the MAPK family triggers the activation of transcription factors such as the nuclear

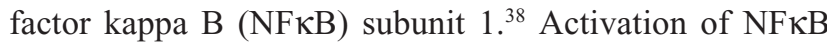
eventually results in the expression of a battery of genes that regulate inflammatory, apoptotic, and proliferative responses associated with the pathogenesis of emphysema. ${ }^{39}$ Previous studies have shown the activation of $\mathrm{NF \kappa B}$ in the bronchial biopsies and inflammatory cells of non-AATD
Table 6 miRNA-target interactions between differentially expressed miRNAs and mRNA in severe vs mild AATD emphysema patients

\begin{tabular}{llll}
\hline $\begin{array}{l}\text { Downregulated } \\
\text { miRNA ID }\end{array}$ & $\begin{array}{l}\text { Upregulated } \\
\text { target gene }\end{array}$ & P-value & logFC \\
\hline hsa-miR-335-5p & EREG & 0.018 & 2.2 \\
& OLFM4 & 0.003 & 1.4 \\
& NR4A2 & 0.004 & 1.2 \\
& JUP & 0.008 & 1.2 \\
& SLC2A3 & 0.012 & 0.97 \\
& NR4AI & 0.018 & 0.97 \\
& NFIL3 & 0.001 & 0.84 \\
& STI4 & 0.002 & 0.73 \\
& TRIBI & 0.015 & 0.70 \\
& RNFI57 & 0.002 & 0.63 \\
& EGR3 & 0.004 & 0.60 \\
Upregulated & MAFB & 0.018 & 0.60 \\
miRNA ID & Downregulated & & \\
hsa-miR-874-3p & target gene & & \\
hsa-miR-193b-3p & HISTIH4H & 0.001 & -1.2 \\
\hline HIST2H3D & 0.002 & -0.6 \\
\hline
\end{tabular}

Notes: Integration of validated and predicted interactions between miRNAs and their target genes using multiMiR $\mathrm{R}$ package. Comparisons were performed using a $P$-value $<0.05$ and $F C> \pm 1.5(\log F C> \pm 0.58)$.

Abbreviations: AATD, alphal-antitrypsin deficiency; logFC, fold change logarithm; miRNA, microRNA.

COPD patients. ${ }^{40}$ Gene expression profiling studies have revealed that inflammatory cytokine- and chemokine-related genes (ie, fractalkine/CX3CL1) are significantly increased in stable non-AATD-COPD patients, becoming more pronounced during COPD exacerbations. ${ }^{21,41}$

Transcripts involved in the JNK cascade pathway were also upregulated in severe compared to mild COPD patients. Recent data by Pastore et $\mathrm{al}^{42}$ revealed that JNK and c-JUN play a role in SERPINA1 gene transcriptional upregulation in AATD-related liver disease. To date, however, there are no studies investigating the impact of JNK on the pathogenesis of AATD.

Changes in miRNA expression are associated with the development and progression of cancer, and cardiovascular and inflammatory pulmonary diseases. ${ }^{13,43}$ We found 8 miRNAs to be downregulated in PBMCs from severe patients, including hsa-miR-486-3p and hsa-miR-335-5p, which have previously been reported to be related to respiratory diseases. Downregulation of hsa-miR-335-5p involves the activation of pathways related to inflammation and angiogenesis. Therefore, our analysis suggests a potential novel link between decreased miR-335-5p expression and the severity of AATD-related emphysema. The integrative analysis revealed that miR-335-5p interacts with other miRNAs previously described in emphysema. For example, high expression of hsa-miR 146-5p in cells from severe 
A

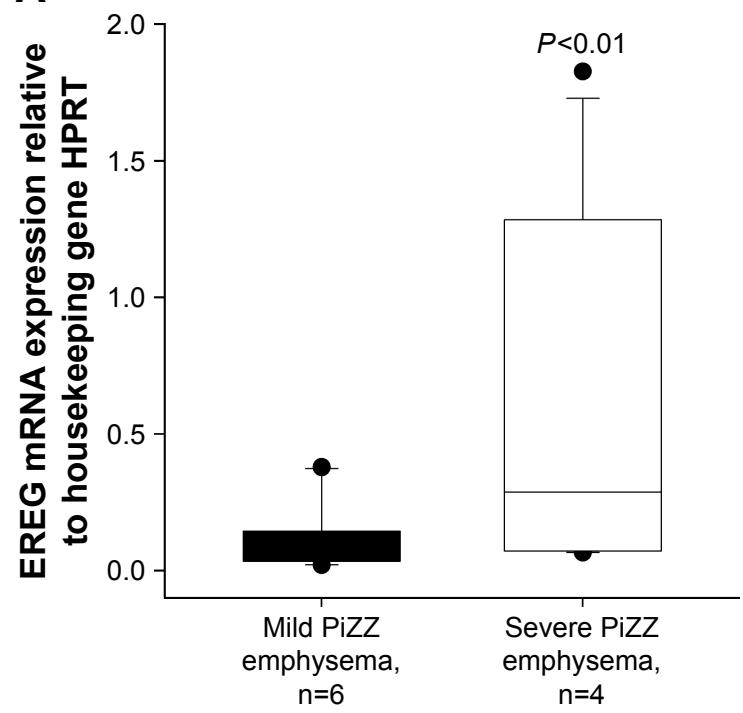

B

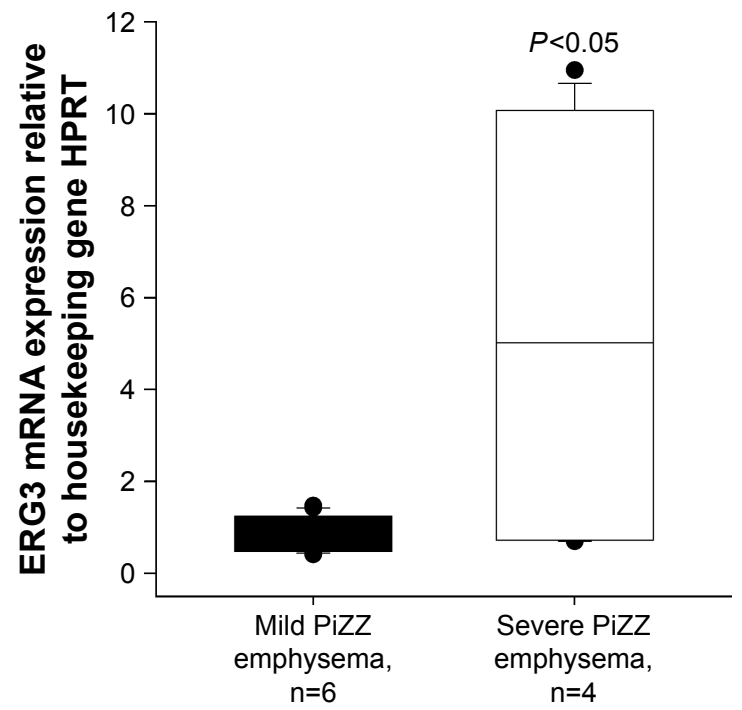

Figure 2 Quantitative reverse transcription polymerase chain reaction.

Note: Validation of EREG (A) and EGR3 (B) expression compared with housekeeping gene hypoxanthine phosphoribosyl transferase (HPRT).

Abbreviation: mRNA, messenger RNA.

patients supports the findings by Ezzie et al, ${ }^{18}$ who stated that miR-146a expression is higher in lung tissue from patients with COPD than in non-COPD smokers. In contrast, Sato et $\mathrm{al}^{44}$ reported reduced expression of miR-146a in cultured fibroblasts from COPD subjects. miR-146a was downregulated in sputum of patients with COPD and smokers compared with never-smokers, and in smokers with COPD in comparison to smokers without COPD. ${ }^{45}$ Overall, recent studies support the hypothesis that miR-146a and miR-146b play a role in the regulation of inflammatory pulmonary disorders. ${ }^{46}$

The results of the present study provide additional support for the role of miRNAs in COPD and improve the understanding of the molecular mechanisms underlying the expression of peripheral inflammatory cells in patients with severe and mild AATD-related emphysema.

\section{Limitations}

We found significant differences in the expression patterns of genes between severe and mild emphysema patients with PiZZ AATD. However, the results from this small pilot study must be complemented by the inclusion of a control group of patients with non-AATD-related COPD. A control group would help to clarify whether the activation of pathways related to inflammation, immune response, and angiogenesis are the cause or the effect of more progressed emphysema. Golpon et $\mathrm{al}^{35}$ found some degree of shared gene profiles between "usual" non-AATD-related emphysema and AATD-related emphysema. These authors observed statistically significant differences in the modulation of genes associated with protein and energy metabolism and immune function, which allow differentiation between these 2 emphysema types at the lung tissue level. We are aware that differences in the genetic signatures of PBMCs isolated from severe vs mild PiZZ AATD patients need to be validated in a larger study population.

In our analysis, 12 samples were initially included, but 2 current smokers were excluded from the patient group with severe AATD emphysema. According to the quality control analysis, the gene expression profile of these individuals was markedly different compared with the other samples. Cigarette smoke is a complex mixture of $>4,000$ chemical compounds that can induce a variety of biological responses, including oxidative stress, apoptosis, and inflammation, among others. ${ }^{47}$ In general, most studies report a global downregulation and upregulation of miRNA and mRNA abundance in response to cigarette smoking. ${ }^{18,48}$ We plan to perform a separate study to analyze the gene expression profiles in PBMCs isolated from smokers with severe and mild COPD.

The cellular gene expression pattern may be influenced by multiple factors, such as patient age, gender, genetic background, and treatment. Replacement therapy could have influenced gene expression. Some studies have suggested that replacement therapy may reduce airway inflammation. ${ }^{49-51}$ However, Olfert et $\mathrm{al}^{52}$ found that replacement therapy 
reduces the concentration of TNF-alpha, but has no effect on IL-6, IL1B, or C-reactive protein. In our study, 4 severe and 2 mild emphysema AATD patients received augmentation therapy every 15 days. To minimize the potential effect of replacement therapy, blood samples were taken just before the patients received the next infusion. However, it would be important to include PBMCs from patients who have never received augmentation therapy.

\section{Conclusion}

The findings of this analysis provide novel information about the different gene profile of PBMCs isolated from patients with severe and mild emphysema with PiZZ AATD. We specifically identified downregulated miRNA (hsa-miR335 ) in severe AATD patients. The hsa-miR-335-5p regulates important transcription factors involved in the activation of pathways directly related to inflammation and angiogenesis and could play an important role in the pathogenesis of this rare condition. Nevertheless, further analyses are needed to validate these results.

\section{Acknowledgments}

The authors would like to thank the Biobank at the Vall d'Hebron Research Institute (VHIR), Barcelona, Spain. This study was funded by a grant from the Fundació Catalana de Pneumologia (FUCAP) year 2017 and by an unrestricted grant from the Catalan Center of Excellence for the management of alpha1-antitrypsin deficiency in the Hospital Universitari Vall d'Hebron (Barcelona) supported by Grifols (Barcelona, Spain).

\section{Disclosure}

The authors report no conflicts of interest in this work.

\section{References}

1. Lozano R, Naghavi M, Foreman K, et al. Global and regional mortality from 235 causes of death for 20 age groups in 1990 and 2010: a systematic analysis for the Global Burden of Disease Study 2010. Lancet. 2012;380(9859):2095-2128.

2. Lomas DA, Mahadeva R. Alpha-1-antitrypsin polymerization and the serpinopathies: pathobiology and prospects for therapy. $J$ Clin Invest. 2002;110(11):1585-1590.

3. DeMeo DL, Silverman EK. Alpha 1-antitrypsin deficiency. 2: genetic aspects of alpha(1)-antitrypsin deficiency: phenotypes and genetic modifiers of emphysema risk. Thorax. 2004;59(3):259-264.

4. Blanco I, Bueno P, Diego I, et al. Alpha-1 antitrypsin Pi*Z gene frequency and Pi*ZZ genotype numbers worldwide: an update. Int J Chron Obstruct Pulmon Dis. 2017;12:561-569.

5. Tirado-Conde G, Lara B, Casas F, et al. Factors associated with the evolution of lung function in patients with alpha-1 antitrypsin deficiency in the Spanish registry. Arch Bronconeumol. 2011;47(10):495-503.

6. Tanash HA, Nilsson PM, Nilsson JA, Pituilainen E. Clinical course and prognosis of never smokers with severe AATD (PiZ). Thorax. 2008; 63(12):1091-1095.
7. The Alpha-1-antitrypsin deficiency Registry Study Group. Survival and $\mathrm{FEV}_{1}$ decline in individuals with severe deficiency of alpha-1antitrypsin. Am J Respir Crit Care Med. 1998;158(1):49-59.

8. Seersholm N, Kok-Jensen A, Dirksen A. Decline in FEV among patients with severe hereditary ATAD type PiZ. Am J Respir Crit Care Med. 1995;152(6 Pt 1):1922-1925.

9. Chen W, Hong YQ, Meng ZL. Bioinformatics analysis of molecular mechanisms of chronic obstructive pulmonary disease. Eur Rev Med Pharmacol Sci. 2014;18(23):3557-3563.

10. Wei L, Xu D, Qian Y, et al. Comprehensive analysis of gene-expression profile in chronic obstructive pulmonary disease. Int JChron Obstruct Pulmon Dis. 2015;10:1103-1109.

11. Faner R, Cruz T, Casserras T, et al. Network analysis of lung transcriptomics reveals a distinct B-Cell signature in emphysema. Am J Respir Crit Care Med. 2016;193(11):1242-1253.

12. Friedman RC, Farh KK, Burge CB, Bartel DP. Most mammalian mRNAs are conserved targets of microRNAs. Genome Res. 2009; 19(1):92-105.

13. Angulo M, Lecuona E, Sznajder JI: Role of microRNAs in lung disease. Arch Bronconeumol. 2012;48(9):325-330.

14. Nana-Sinkam SP, Hunter MG, Nuovo GJ, et al. Integrating the MicroRNome into the study of lung disease. Am J Respir Crit Care Med. 2009; 179(1):4-10

15. Tomankova T, Petrek M, Kriegova E. Involvement of microRNAs in physiological and pathological processes in the lung. Respir Res. 2010;11:159.

16. Pagdin T, Lavender P. MicroRNAs in lung diseases. Thorax. 2012; 67:183-184

17. Raponi M, Dossey L, Jatkoe T, et al. MicroRNA classifiers for predicting prognosis of squamous cell lung cancer. Cancer Res. 2009; 69(14):5776-5783.

18. Ezzie ME, Crawford M, Cho JH, et al. Gene expression networks in COPD: microRNA and mRNA regulation. Thorax. 2012;67(2): $122-131$.

19. Hobbs BD, Hersh CP. Integrative genomics of chronic obstructive pulmonary disease. Biochem Biophys Res Commun. 2014;452(2): 276-286.

20. Chen ZH, Kim HP, Ryter SW, Choi AM. Identifying targets for COPD treatment through gene expression analyses. Int $J$ Chron Obstruct Pulmon Dis. 2008;3(3):359-370.

21. Wu X, Sun X, Chen C, Bai C, Wang X. Dynamic gene expressions of peripheral blood mononuclear cells in patients with acute exacerbation of chronic obstructive pulmonary disease: a preliminary study. Crit Care. 2014;18(6):508.

22. American Thoracic Society/European Respiratory Statement: Standards for the diagnosis and management of individuals with alpha-1 antitrypsin deficiency. Am J Respir Crit Care Med. 2003;168(7):818-900.

23. Aggarwal N, Koepke J, Matamala N, et al. Alpha-1 antitrypsin regulates transcriptional levels of serine proteases in blood mononuclear cells. Am J Respir Crit Care Med. 2016;193(9):1065-1067.

24. Miravitlles M, Soler-Cataluña JJ, Calle M, et al. Spanish COPD guidelines (GesEPOC) 2017. Pharmacological treatment of stable chronic obstructive pulmonary disease. Arch Bronconeumol. 2017;53(6): 324-335.

25. Casas F, Blanco I, Martínez MT, et al. Indications for active case searches and intravenous alpha-1 antitrypsin treatment for patients with alpha-1 antitrypsin deficiency chronic obstructive pulmonary disease: an update. Arch Bronconeumol. 2015;51(4):185-192.

26. Carvalho BS, Irizarry RA. A framework for oligonucleotide microarray preprocessing. Bioinformatics. 2010;26(19):2363-2367.

27. Irizarry RA, Hobbs B, Collin F, et al. Exploration, normalization, and summaries of high density oligonucleotide array probe level data. Biostatistics. 2003;4(2):249-264.

28. Leek JT, Johnson WE, Parker HS, et al. (2016). sva: Surrogate Variable Analysis. R package version 3.20.0. Available from: https:// bioconductor.riken.jp/packages/3.0/bioc/html/sva.html. Accessed October 18, 2017. 
29. Johnson WE, Li C, Rabinovic A. Adjusting batch effects in microarray expression data using empirical Bayes methods. Biostatistics. 2007; $8(1): 118-127$

30. Smyth GK. Linear models and empirical Bayes methods for assessing differential expression in microarray experiments. Stat Appl Genet Mol Biol. 2004;3:Article3

31. Ashburner M, Ball CA, Blake JA, et al. Gene ontology: tool for the unification of biology. the gene ontology consortium. Nat Genet. 2000;25(1):25-29.

32. Ru Y, Kechris KJ, Tabakoff B, et al. The multiMiR R package and database: integration of microRNA-target interactions along with their disease and drug associations. Nucleic Acids Res. 2014;42(17):e133.

33. Agusti AG, Noguera A, Sauleda J, Sala E, Pons J, Busquets X. Systemic effects of chronic obstructive pulmonary disease. Eur Respir J. 2003;21(2):347-360.

34. Janciauskiene SM, Bals R, Koczulla R, Vogelmeier C, Köhnlein T, Welte $\mathrm{T}$. The discovery of $\alpha 1$-antitrypsin and its role in health and disease. Respir Med. 2011;105(8):1129-1139.

35. Golpon HA, Coldren CD, Zamora MR, et al. Emphysema lung tissue gene expression profiling. Am J Respir Cell Mol Biol. 2004;31(6): 595-600.

36. Lee SL, Sadovsky Y, Swirnoff AH, et al. Luteinizing hormone deficiency and female infertility in mice lacking the transcription factor NGFI-A (Egr-1). Science. 1996;273:1219-1221.

37. Baron VT, Pio R, Jia Z, Mercola D. Early growth response 3 regulates genes of inflammation and directly activates IL6 and IL8 expression in prostate cancer. Br J Cancer. 2015;112(4):755-764.

38. Underwood DC, Osborn RR, Bochnowicz S, et al. SB 239063, a p38 MAPK inhibitor, reduces neutrophilia, inflammatory cytokines, MMP-9, and fibrosis in lung. Am J Physiol Lung Cell Mol Physiol. 2000; 279(5):L895-L902.

39. Johnson GL, Lapadat R. Mitogen-activated protein kinase pathways mediated by ERK, JNK, and p38 protein kinases. Science. 2002;298(5600):1911-1912.

40. Schuliga M. NF-KappaB in chronic inflammatory airway disease. Biomolecules. 2015;5(3):1266-1283.

41. Yamamoto C, Yoneda T, Yoshikawa M, et al. Airway inflammation in COPD assessed by sputum levels of interleukin-8. Chest. 1997;112(2): 505-510.
42. Pastore N, Attanasio S, Granese B, et al. Activation of the c-Jun $\mathrm{N}$-terminal kinase pathway aggravates proteotoxicity of hepatic mutant Z alpha1-antitrypsin. Hepatology. 2017;65(6):1865-1874.

43. Huang JT, Wang J, Srivastava V, Sen S, Liu SM. MicroRNA machinery genes as novel biomarkers for cancer. Front Oncol. 2014;4:113.

44. Sato T, Liu X, Nelson A, et al. Reduced MiR-146a increases prostaglandin E2 in chronic obstructive pulmonary disease fibroblasts. Am J Respir Crit Care Med. 2010;182(8):1020-1029.

45. Van Pottelberge GR, Mestdagh P, Bracke KR, et al. MicroRNA expression in induced sputum of smokers and patients with chronic obstructive pulmonary disease. Am J Respir Crit Care Med. 2011;183(7): 898-906.

46. Tomankova T, Petrek M, Kriegova E. Involvement of microRNAs in physiological and pathological processes in the lung. Respir Res. 2010;11:159.

47. Moretto N, Volpi G, Pastore F, Facchinetti F. Acrolein effects in pulmonary cells: relevance to chronic obstructive pulmonary disease. Ann NY Acad Sci. 2012;1259:39-46.

48. Graff JW, Powers LS, Dickson AM, et al. Cigarette smoking decreases global microRNA expression in human alveolar macrophages. PLoS One. 2012;7(8):e44066.

49. Wang Y, He Y, Abraham B, et al. Cystosolic, autocrine alpha-1 proteinase inhibitor (A1P1) inhibits caspase-1 blocks IL-1 beta dependent cytokine release in monocytes. PLoS One. 2012;7(11):e51078.

50. Stockley RA, Bayley DL, Unsal I, Dowson LJ. The effect of augmentation therapy on bronchial inflammation in alphal antitrypsin deficiency. Am J Respir Crit Care Med. 2002;165(11):1494-1498.

51. Schimid ST, Koepke J, Dresel M, et al. The effects of weekly augmentation therapy in patients with PiZZ alpha-1 antitrypsin deficiency. Int $J$ Chron Obstruct Pulmon Dis. 2012;7:687-696.

52. Olfert IM, Malek MH, Eagan TM, Wagner H, Wagner PD. Inflammatory cytokine response to exercise in alpha-1-antitrypsin deficient COPD patients 'on' or 'off' augmentation therapy. BMC Pulm Med. 2014;14:106.
International Journal of COPD

\section{Publish your work in this journal}

The International Journal of COPD is an international, peer-reviewed journal of therapeutics and pharmacology focusing on concise rapid reporting of clinical studies and reviews in COPD. Special focus is given to the pathophysiological processes underlying the disease, intervention programs, patient focused education, and self management protocols.

\section{Dovepress}

This journal is indexed on PubMed Central, MedLine and CAS. The manuscript management system is completely online and includes a very quick and fair peer-review system, which is all easy to use. Visit http://www.dovepress.com/testimonials.php to read real quotes from published authors. 